\title{
An MMG-based Human-Assisting Manipulator Using Acceleration Sensors
}

\author{
Keisuke Shima and Toshio Tsuji \\ Graduate School of Engineering \\ Hiroshima University \\ 1-4-1 Kagamiyama, Higashi-hiroshima, Japan \\ \{shima, tsuji\}@bsys.hiroshima-u.ac.jp
}

\begin{abstract}
This paper proposes a control method for a humanassisting manipulator using acceleration sensors. The technique involves an arm control part (ACP) and a hand-and-wrist control part (HWCP); the ACP controls the manipulator's shoulder and elbow joints using acceleration signals, while the HWCP controls the corresponding joints using mechanomyogram (MMG) signals measured from the human operator. A distinctive feature of the proposed method is its estimation of information on force and motion from measured acceleration signals using MMG processing and a probabilistic neural network. Experiments demonstrated that the MMG patterns seen during hand and wrist motion can be classified sufficiently (average rate: $94.3 \%$ ), and that a prosthetic manipulator can be controlled using the acceleration signals measured. Such manipulators are expected to prove useful as assistive devices for people with physical disabilities.
\end{abstract}

Index Terms-Mechanomyogram, pattern classification, probabilistic neural network, human-machine interface

\section{INTRODUCTION}

Electromyogram (EMG) detection is the most commonly utilized means of interaction between amputees and externally powered prostheses [1]-[5]. Since EMG signals change depending on the level of muscular contraction, these externally powered units can be controlled in a natural way.

Using EMG signals, Fukuda et al. [6] developed a humanassisting manipulator consisting of a forearm part and an upper-arm part. The forearm part can be controlled using EMG electrodes attached to the skin surface, while the upper-arm part uses a $3 \mathrm{D}$ position sensor attached to the end point of the arm. Although this method allows the operator to control the manipulator at will, it requires the combination of two kinds of sensors. It is also quite difficult to use the manipulator for long periods of time because EMG signals are easily affected by changes in the skin's impedance.

Meanwhile, some utilization of mechanomyogram (MMG) [7] signals has been explored [8], [9]. These signals express the mechanical vibration of muscles, and lie between EMG signals - which express electrical activity in muscles - and the resulting muscle force. Therefore, in the same way as their EMG counterparts, MMG signals change according to the level of muscular contraction [10], [11]. It should be noted that MMG signals are not affected by changes in skin impedance caused by sweating [8]. Barry et al. [8] and Silva et al. [9] achieved on/off control of a prosthetic hand by ascertaining the presence or absence of muscular contraction. However,

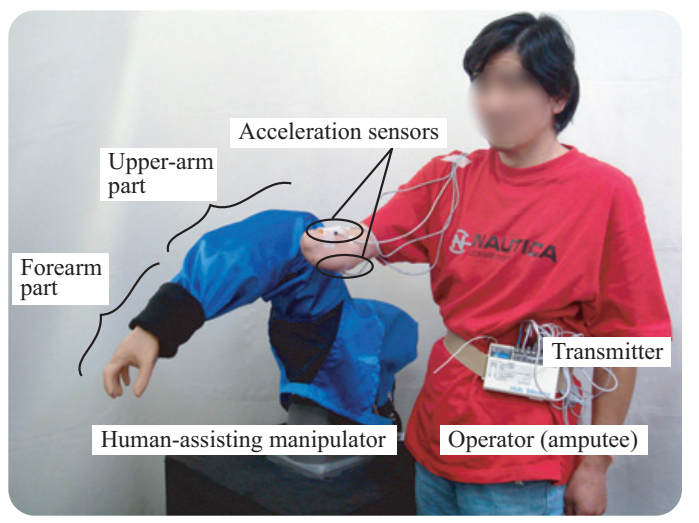

Fig. 1. Example of human-assisting manipulator operation by an amputee

these studies cannot be extended to proportional control of multifunctional prostheses using MMG signals because classification of multiple motions and estimation of muscular force from the measured signals are necessary.

In this paper, we propose a novel control method for a multifunctional human-assisting manipulator based on motion classification of MMG signals using a probabilistic neural network. First, from the signals measured by the acceleration sensors, two kinds of biological signals - MMG and motion acceleration (MAC) - are estimated using digital filters. The operator's intended forearm/hand motion is then estimated from the MMG signals, which are utilized to proportionally control the forearm part of the manipulator. The MAC signals are also used to control the upper-arm part of the manipulator after being integrated twice. As a result, it is possible to control the manipulator using acceleration sensors only.

This paper is organized as follows: in Section II, the details of the proposed method are described. The validity of the proposed system is examined in Section III. Finally, the paper is concluded in Section IV, and future study plans are discussed.

\section{Manipulator CONTROL USing ACCELERATion SIGNALS}

Figure 1 shows an example involving a forearm amputee using the proposed method to operate a human-assisting manipulator. The manipulator [6] consists of a forearm part that 


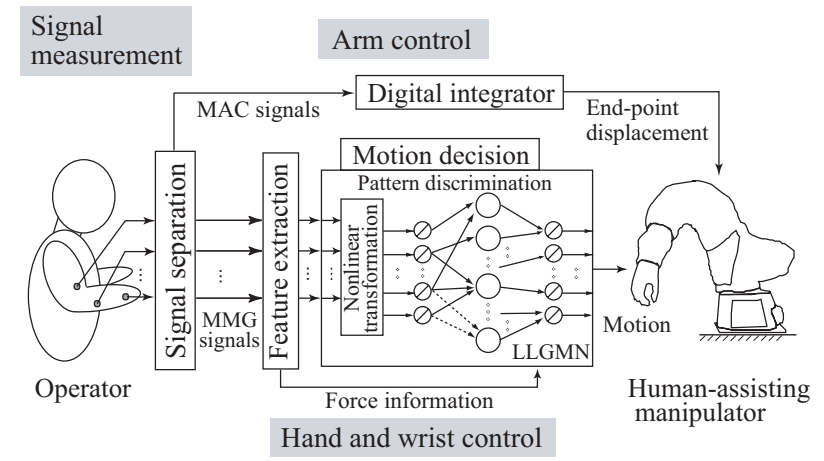

Fig. 2. The proposed human-assisting manipulator control method

uses three ultrasonic motors with one degree of freedom (DOF) each, while the upper-arm part uses a four-DOF robot arm (Mitsubishi Electric Corporation). The forearm part can also be used as a prosthetic hand attached to the amputated part.

An outline of the proposed control method for humanassisting manipulators is shown in Fig 2. The technique consists of three parts: signal measurement, hand and wrist control and arm control. In the signal measurement part, the measured signals are separated into MMG and MAC types. The operator's intended motion is estimated from the MMG signals for the hand and wrist control part (HWCP), and the forearm part of the manipulator (the prosthetic hand) is then controlled using the estimated results. The arm control part (ACP) computes the three-dimensional motion of the operator's arm from the MAC signals, and the upper arm of the manipulator is then controlled to track the desired trajectory. The details of each control part are outlined in the following subsections.

\section{A. Signal measurement part}

First, acceleration signals are measured from $L$ channels of acceleration sensors attached to the muscle surface on the forearm. Although the measured signals contain information on motion acceleration (MAC) in addition to the MMG, the frequency components of the MMG are higher [11] than those of MAC. Accordingly, the two components can be separated in the frequency region using a set of digital filters with appropriate properties.

In this paper, the two types of digital band-pass filter used for signal separation are a low-band filter $\left(f_{A l} \mathrm{~Hz}\right.$ to $\left.f_{A h} \mathrm{~Hz}\right)$ for MAC signals and a high-band filter $\left(f_{M l} \mathrm{~Hz}\right.$ to $\left.f_{M h} \mathrm{~Hz}\right)$ for the MMG signals. After signal separation, the $t$ th samples of MAC and MMG signals are defined as $M A C_{l}(t)(l=$ $1,2, \ldots, L)$ and $M M G_{l}(t)$ at a given time $t$.

Since MMG and MAC signals reflect muscle activation and information on physical movement, respectively, these data are used to control each part of the manipulator.

\section{B. Hand and wrist control part (HWCP)}

In the HWCP, the operator's intended motion and force information are estimated from the MMG signals separated from the acceleration signals, and the forearm part of the manipulator is controlled in line with the estimated results.

1) Feature extraction: The MMG signals are rectified and smoothed through a second-order low-pass Butterworth filter (cutoff frequency: $f_{\text {cut }} \mathrm{Hz}$ ). The filtered signals are then normalized to make the sum of $L$ channels equal to 1 , and are defined as the feature vector $\boldsymbol{x}(t)=\left[x_{1}(t), x_{2}(t), \cdots, x_{L}(t)\right]^{\mathrm{T}} \in$ $\Re^{L}$ at a given time $t$. Here, each element of the feature vector $x_{i}(t)(i=1, \cdots, L)$ is defined as

$$
x_{i}(t)=\frac{M M G_{i}(t)-M M G_{i}^{s t}}{\sum_{i^{\prime}=1}^{L}\left(M M G_{i^{\prime}}(t)-M M G_{i^{\prime}}^{s t}\right)},
$$

where $M M G_{i}^{s t}$ is the mean value of $M M G_{i}(t)$, which is measured while the muscles are relaxed. The feature vector $\boldsymbol{x}(t)$ is input into the probabilistic neural network for estimation of the operator's intended motion.

Since the amplitude level of MMG signals is known to change in proportion to muscle force, the operator's muscle contraction level can be estimated from these signals for manipulator control. First, the root mean square (RMS) of the MMG signals is computed, and then the value is normalized using the root mean square MMG with maximum voluntary contraction (MVC). The average of $L$ normalized signals is also used to compute force information $F_{M M G}(t)$ as follows:

$$
\begin{aligned}
F_{M M G}(t) & =\frac{1}{L} \sum_{i=1}^{L} \frac{R M S_{i}(t)-R M S_{i}^{s t}}{R M S_{i}^{\text {max }}-R M S_{i}^{s t}} \\
R M S_{i}(t) & =\sqrt{\frac{1}{n} \sum_{\tau=0}^{n-1} M M G_{i}(t-\tau)^{2}},
\end{aligned}
$$

where $n$ is the number of RMS values, $R M S_{i}^{\max }$ is the mean value of $R M S_{i}(t)$ at the MVC of the muscle, and $R M S_{i}^{s t}$ is the mean value of $R M S_{i}(t)$, which is measured while the muscles are relaxed. The motors of the forearm part are operated in by the level of force information $F_{M M G}(t)$ using the impedance control method [6].

2) $M M G$ pattern classification: The feature vector extracted from the MMG signals is classified by a probabilistic neural network (PNN), and the operator's intended motion is estimated. In the proposed method, the log-linearized Gaussian mixture network (LLGMN) [12] proposed by Tsuji et al. is used as the PNN for the discrimination of MMG patterns. The LLGMN is based on a Gaussian mixture model (GMM), and can estimate the probability density function (pdf) from the input vector through learning. The LLGMN has been utilized as a classification method for various biological signals such as EMGs and electroencephalograms (EEGs) with a high classification rate [6], [12].

In the proposed method, the system first measures $M$ types of MMG patterns during motion conducted by an operator, such as hand grasping and opening. The feature vectors calculated from these MMG signals are then input to the LLGMN as teacher vectors, and the LLGMN is trained to estimate the a posteriori probabilities of each motion. After 
learning, the a posteriori probability of each learned motion $Y^{m}(t)(m=1, \cdots, M)$ can be output for each input vector. According to the learning ability of the LLGMN, it is possible for it to correspond to changes in MMG signals stemming from differences among individuals.

For motion classification, the force information $F_{M M G}(t)$ is evaluated using the determination threshold $F_{t h}$. When $F_{M M G}(t)$ exceeds $F_{t h}$, the system judges the occurrence of operator motion, and the feature vector is input to the LLGMN. At the same time, in order to prevent discrimination errors, we calculate the entropy $H(t)$, defined as

$$
H(t)=-\sum_{m=1}^{M} Y^{m}(t) \log _{2} Y^{m}(t)
$$

If $H(t)$ is smaller than the discrimination determination threshold $H_{t h}$, the motion with the highest a posteriori probability becomes the result of discrimination. Otherwise, if $H(t)$ exceeds $H_{t h}$, discrimination is suspended as obscure motion since large entropy means that the LLGMN output is ambiguous. When this happens, the LLGMN outputs the same result as one sample before to the manipulator. In addition, when and only when the same discrimination results are given $T i_{s t b}$ times repeatedly, the manipulator is controlled in order to prevent manipulator malfunction. Further, the driving speed of the HWCP is controlled in proportion to the force information $F_{M M G}(t)$.

\section{Arm control part (ACP)}

In the ACP, the MAC signals separated from the acceleration signals are integrated twice, and hand displacement is then estimated from these signals. The manipulator's end-point is then moved to the target position computed. In order to remove the influence of the measurement bias of the acceleration sensors, the value of a single integrated MAC signal is defined as $V_{\text {int }}$, and the value of velocity $V(t)$ is calculated using the following equation:

$$
V(t)=F\left[V_{\text {int }}(t)-V_{f i l}\left(t-t_{d e l}\right)\right],
$$

where $V_{f i l}(t)$ is the smoothed $V_{\text {int }}(t)$ through a second-order low-pass filter (cutoff frequency: $f_{\text {int }} \mathrm{Hz}$ ), and $t_{\text {del }}$ describes the phase-lag element of the filter. $F[x]$ represents a function with the dead zone expressed as follows:

$$
F[x]=\left\{\begin{array}{ll}
x-V_{t h} & \left(x>V_{t h}\right) \\
0 & \left(|x| \leq V_{t h}\right) \\
x+V_{t h} & \left(x<-V_{t h}\right)
\end{array} .\right.
$$

$V(t)$ is then integrated twice using trapezoidal integration, and the displacement $P(t)$ is calculated to control the end-point displacement of the manipulator.

The desired values for the joint angles of the manipulator's upper arm are then determined according to the end-point displacement calculated, and the corresponding joints are controlled using a proportional-integral-derivative (PID) control method.
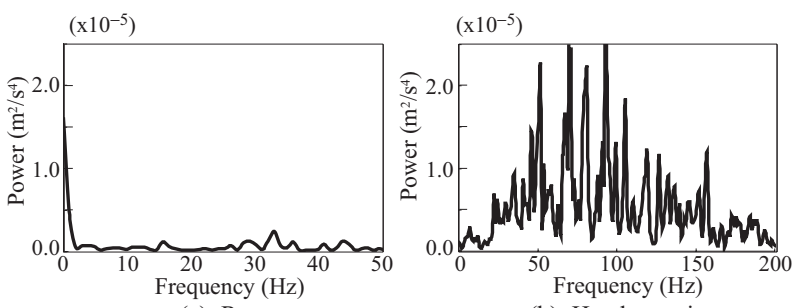

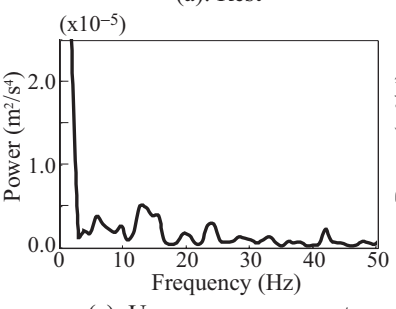

(c): Upper-arm movements

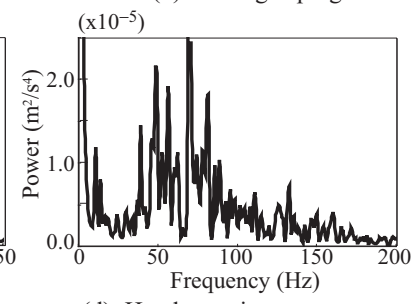

(d): Hand grasping with upper-arm movements
Fig. 3. Power spectra of the measured acceleration signals (subject A)

\section{EXPERIMENTS}

To demonstrate and verify the validity of the proposed method, we conducted experiments with four normal subjects (male; A, B: age 24; C, D: age 23) and a forearm amputee (male; E: age 49). One triaxial acceleration sensor (Nihon Kohden Corporation, TA-513 G: rectangular parallelepiped, longitudinal $20.0 \mathrm{~mm}$, transversal $15.0 \mathrm{~mm}$, thickness 12.0 $\mathrm{mm}$, weight $12.5 \mathrm{~g}$ ) and three uniaxial acceleration sensors (Nihon Kohden Corporation MT-3 T: wheel, diameter $3.0 \mathrm{~mm}$, thickness $6.5 \mathrm{~mm}$, weight $3.0 \mathrm{~g}$ ) were attached to right forearm of the subjects. The sampling frequency for measuring acceleration signals was $1 \mathrm{kHz}$. Muscular activity was measured from a total of four channels consisting of the $z$-axis of the triaxial acceleration sensor and the three uniaxial acceleration sensors. Elements of upper arm movement were measured from the $x$ ,$y$-, and $z$-axes of the triaxial acceleration sensor. Informed consent was obtained from all subjects.

\section{A. Preliminary experiments}

First, we attempted to separate MAC and MMG signals from the measured signal using the acceleration sensor. A uniaxial acceleration sensor was attached to the skin surface of the right forearm (near the flexor carpi radialis), and the signals of four states - (a) rest, (b) hand grasping, (c) upper-arm movement and (d) hand grasping with upper-arm movement - were measured. We conducted frequency analysis using the FFT (periodogram); the number of data was 2,048, with 512 overlap data. The experimental results are shown in Fig. 3.

Figure 3(a) shows that the elements of the sensor's noise and physiological tremor have low frequency, and (b) indicates that the power spectrum of the MMG signals generated by muscle contraction with hand grasping is included in the range of about 20 to $160 \mathrm{~Hz}$. Fig. 3(c) also indicates that the frequency component of the MAC signals is contained in the range of 


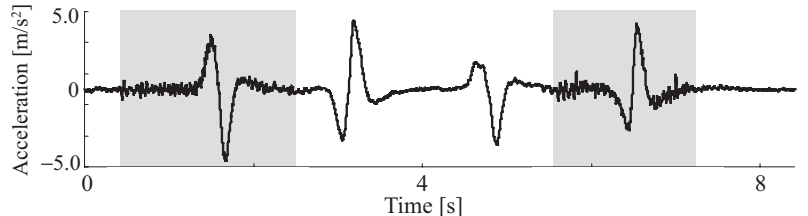

(a) Acceleration signals

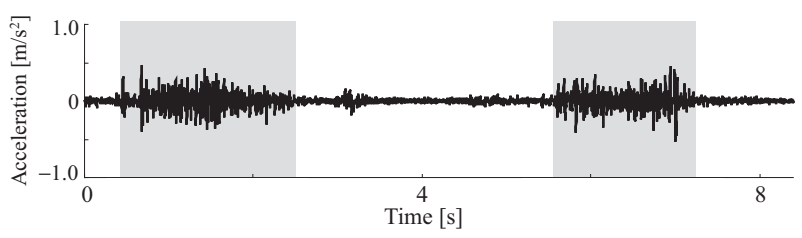

(b) MMG signals

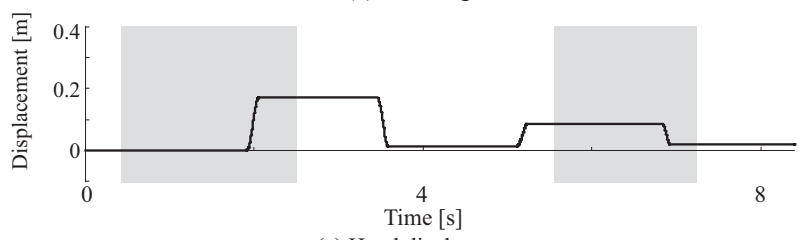

(c) Hand displacement

Fig. 4. Measured and extracted signals (raw acceleration, MMG, and estimated hand displacement)

about 0 to $15 \mathrm{~Hz}$. In addition, Fig. 3(d) shows that the two frequency elements of the MAC and MMG signals can be observed in the low- and high-frequency ranges at the same time. Using a second-order band-pass Butterworth filter, the separated signals are next shown in Fig. 4, in which figures (a) to (c) describe the MAC signals, the MMG signals and the estimated hand displacement, respectively. The parameters of the band-pass filter are set as $f_{M l}=30 \mathrm{~Hz}$ and $f_{M h}=150 \mathrm{~Hz}$ for the separation of the MMG signals. The gray area indicates the time during which the hand is grasping. In the figure, the MAC and MMG signals are generated when the hand is being physically moved and the hand is grasping, respectively, and these signals can be separated using the band-pass filter. Furthermore, hand displacement can be estimated from the MAC signals; we therefore concluded that MMG signals and hand displacement could be measured and extracted as mutually independent.

Moreover, to confirm the MMG characteristics for muscle activation, comparison experiments for EMG and MMG signals were conducted. For the measurement of EMG signals, an EMG electrode was attached to the skin surface of the right forearm (near the flexor carpi radialis, as close as possible to the measurement position of the acceleration signals). Examples of the measured signals are shown in Fig. 5. The gray areas indicate the period during motion (hand grasping). From the figure, it is observed that the MMG signals change with a tendency similar to that of EMG signals. The coefficient of correlation between the smoothed and rectified EMG and the smoothed and rectified MMG is 0.945 . These results indicate that the muscle activation level can be estimated by MMG signals through separation from acceleration signals

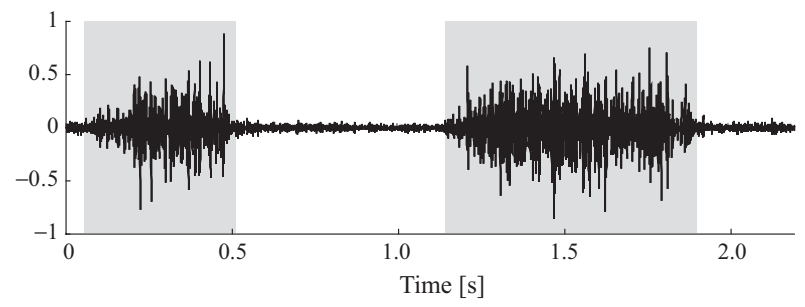

(a) EMG signals

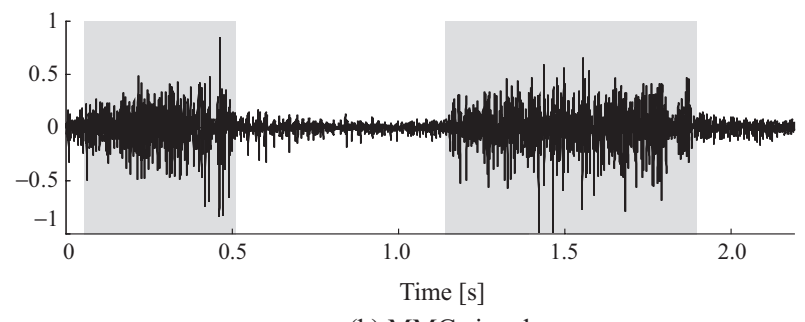

(b) MMG signals

Fig. 5. EMG and MMG signals measured during hand grasping motion

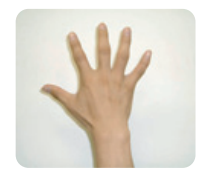

1: Hand opening

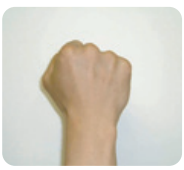

2: Hand grasping

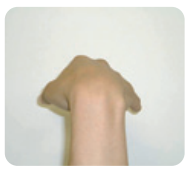

3: Wrist flexion

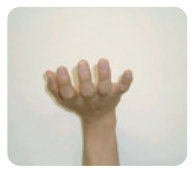

4: Wrist extension
Fig. 6. Forearm motions used in the experiments

with almost the same accuracy as EMG measurement.

\section{B. Estimation of motion}

We conducted experiments to demonstrate that it is possible to estimate the subjects' intended motion with MMG signals and ascertain the direction of hand movement using MAC signals. In these experiments, each subject performed the four motions ( $M=4$; hand opening, grasping, flexion and extension) shown in Fig. 5. The parameters of the band-pass filter for MMG signal separation were set as $f_{M l}=30 \mathrm{~Hz}$ and $f_{M h}=150 \mathrm{~Hz}$, and the MMG signals were smoothed with a low-pass filter cut-off at $f_{\text {cut }}=0.5 \mathrm{~Hz}$ for extraction of the feature vector. In the learning process of the LLGMN, 200 patterns were randomly selected from MMG signals for each motion, and a total of $M \times 200$ patterns were used as teacher vectors. In addition, the threshold $H_{t h}$ was set as 0.4 , $F_{t h}$ as 0.25 , and $T i_{s t b}$ as 400 . In the ACP, the cut-off $f_{\text {int }}$ was set as 0.7 , the phase-lag element $t_{d e l}$ as $0.5 \mathrm{~s}$, and the velocity threshold $V_{t h}$ as $0.2 \mathrm{~m} / \mathrm{s}$. The subjects were asked to conduct linear reciprocating movement with the upper arm and to move along the $y$-axis as precisely as possible.

The experimental results obtained from Subject $\mathrm{E}$ are shown in Fig. 7, which outlines the MAC signals of the triaxial acceleration sensor, the MMG signals of the uniaxial acceleration sensors and triaxial acceleration sensor ( $z$-axis), force infor- 


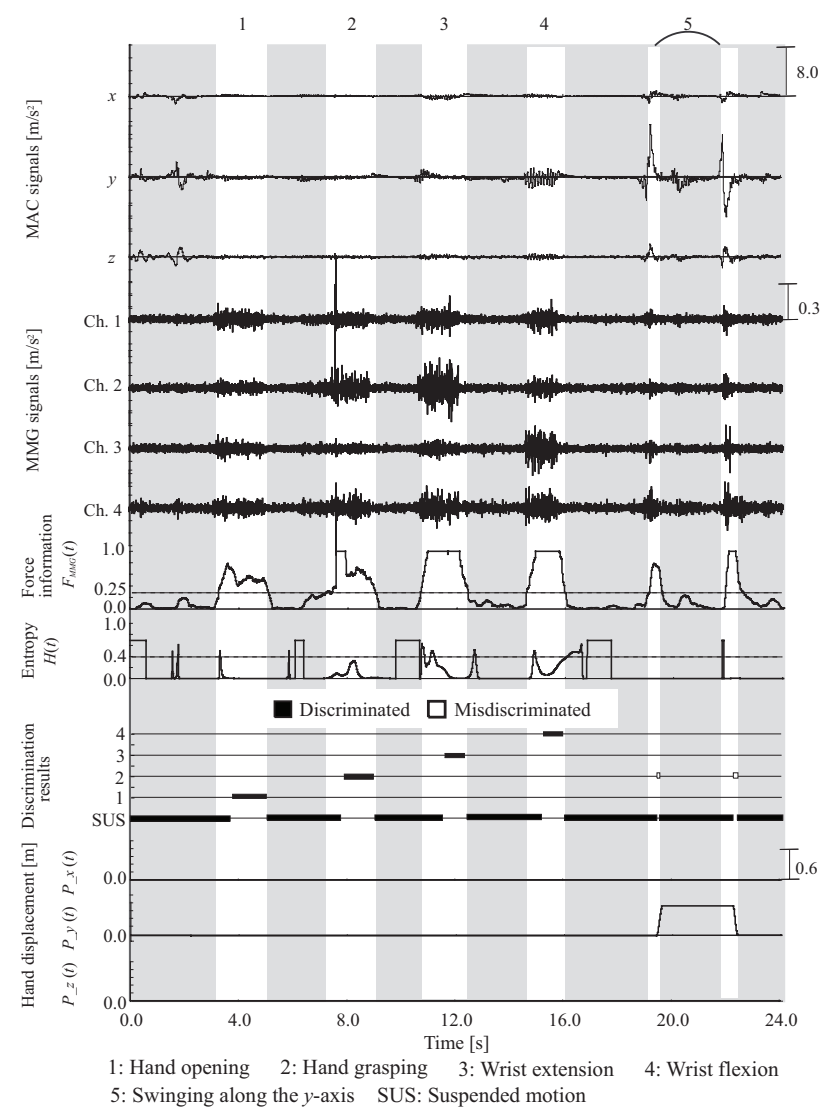

Fig. 7. Classification results using MMG patterns (Subject E)

mation $F_{M M G}(t)$, entropy $H(t)$, the discrimination results, and the estimated hand displacement by the MAC signals. The gray areas indicate that there was no motion because the force information $F_{M M G}(t)$ was below the threshold $F_{t h}$. The MMG patterns seen during each motion are also shown in Fig. 8. The solid lines and shaded areas describe the mean value and standard deviation of the MMG patterns. From these figures, it is observed that different patterns can be measured according to each motion, and these patterns can be classified using the proposed method. Furthermore, Fig. 7 indicates that hand displacement and the hand movement direction can be estimated from the acceleration signals with little time lag during control of the manipulator's upper-arm part.

The average discrimination rates and standard deviation for all subjects' motions are shown in Table I. The number of trials for each motion was five. Here, Subject A had extensive experience in MMG control, while Subjects B E no experience. The table indicates that the discrimination rates were high for each subject, although the beginners' rates were lower than that of Subject A. The average discrimination rate for all the subjects was $94.3 \%$. The experimental results indicate that MMG patterns can be classified correctly and used to estimate operator motion.

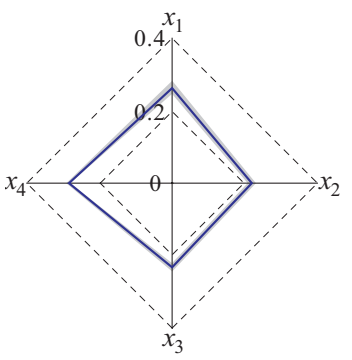

1: Hand opening

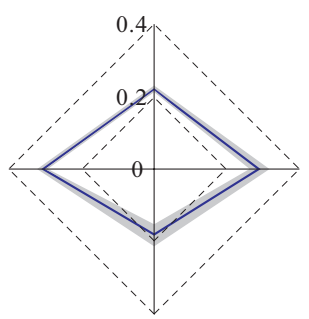

2: Hand grasping

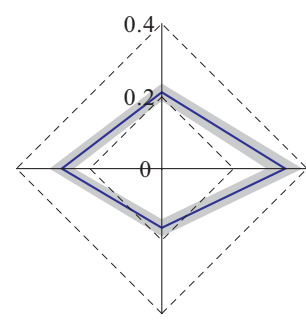

3: Wrist flexion

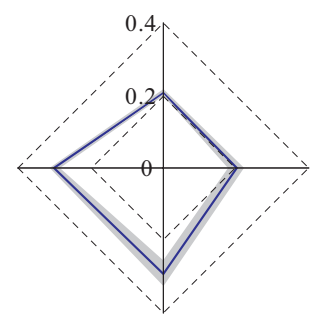

4: Wrist extension
Fig. 8. MMG patterns during forearm motions (subject E)

TABLE I

MOTION DISCRIMINATION RATES

\begin{tabular}{c|c||c|c}
\hline \hline Subject & $\begin{array}{l}\text { Disc. rates } \\
\text { (Ave } \pm \text { SD \%) }\end{array}$ & Subject & $\begin{array}{l}\text { Disc. rates } \\
\text { (Ave } \pm \text { SD \%) }\end{array}$ \\
\hline A & $99.20 \pm 1.23$ & D & $89.64 \pm 2.50$ \\
B & $94.04 \pm 3.42$ & E & $92.38 \pm 7.34$ \\
C & $96.30 \pm 2.61$ & Total & $94.31 \pm 3.42$ \\
\hline \hline
\end{tabular}

\section{Examples of the manipulator control}

To ascertain the effectiveness of the proposed control method, we used it in manipulator control experiments. Figure 9 shows examples of motion pictures taken during manipulator control. Figures 9 (a) and (b) represent control of the forearm part using the MMG signals of an amputee and the upperarm part using the MAC signals of a normal subject. The plotted circles track the trajectories of the triaxial acceleration sensor attached to the subject's forearm and the manipulator's end-point with time interval of 0.1 s. From Fig. 9(a), it is confirmed that the forearm part (prosthetic hand) can be controlled by classifying the amputee's intended motion using MMG signals. Furthermore, it is observed that the upperarm part of the manipulator is moved in response to the arm movement conducted by the subject (Fig. 9(b)). These results lead us to conclude that MMG patterns could be used to estimate the amputee's intended motion, and that MAC signals could be used to determine the large movement of the upperarm conducted by the operator for control of the manipulator's upper-arm part. 

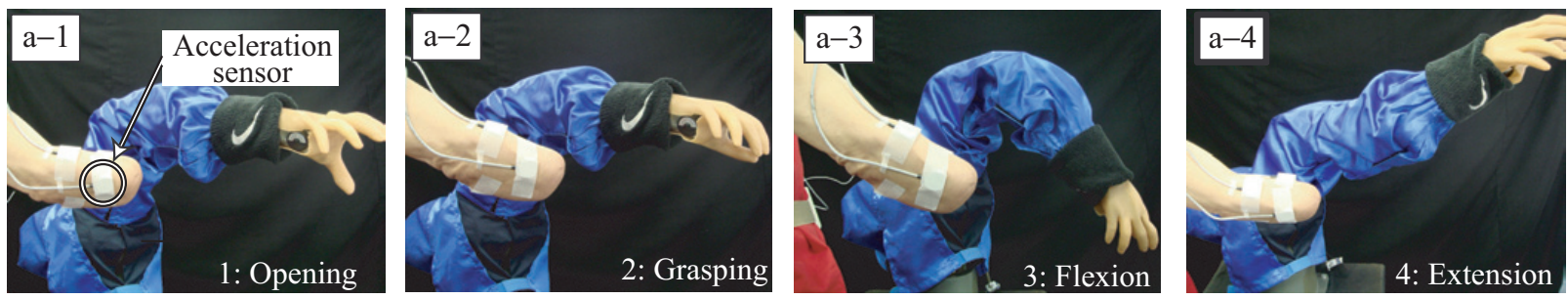

(a) Forearm contol by an amputee
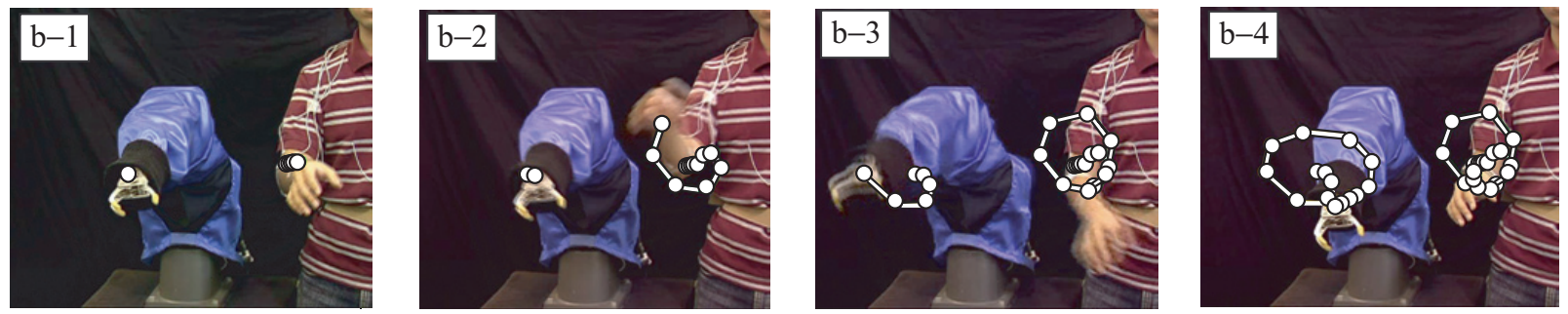

(b) Upper-arm control by a normal subject

Fig. 9. Examples of the motion pictures during manipulator control

\section{CONCLUSION}

This paper proposes a method whereby MMG and MAC signals can be measured at the same time and used as a base control method for human-assisting manipulators. In the experiments performed, an appropriate filter was set for signals measured from acceleration sensors, and it was confirmed that MMG signals (which reflect muscle activity) and MAC signals (which reflect physical movement) could be separated. By discriminating the separated MMG signals using an LLGMN, we also confirmed that the operator's intended motion could be estimated, and showed that the hand displacement of the operator could be estimated from MAC signals measured using acceleration sensors only. Moreover, the acceleration sensors do not have to be attached directly to the skin surface [6]; this means that the measured signals are not affected by changes in skin impedance, and the user can operate the manipulator easily with less stress for long periods.

MMG signal research is a relatively new field, and there are few reports on studies involving interfaces such as the one examined in this paper. In future work, we would like to create an algorithm to enable adaptation to pattern changes resulting from fatigue and long periods of discrimination in order to develop a rehabilitation system using this technique, and to apply the method to other computer interfaces.

\section{ACKNOWLEDGMENT}

The authors would like to express their gratitude to Mr. Tomoyuki Ichiza for his efforts in the experiments. This study was partially supported by the 21st Century COE Program of JSPS (Japan Society for the Promotion of Science) on "Hyper-Human Technology toward the 21st Century Industrial Revolution".

\section{REFERENCES}

[1] N. Wiener, CYBERNETICS or Control and Communication in the Animal and the Machine. Cambrige, MA: MIT Press, 1948.

[2] S. C. Jacobson, D. F. Knutti, R. T. Johonson, and H. H. Sears, "Development of the Utah artificial arm," IEEE Trans. Biomed. Eng., vol. BME-29, pp. 242-269, 1982.

[3] K. Akazawa, H. Takizawa, Y. Hayashi, and K. Fujii, "Development of control system and myoelectric signal processor for biomimetic prosthetic hand," Biomechanism 9, pp. 43-53, 1988.

[4] C. J. Abul-haj and N. Hogan, "Functional assessment of control systems for cybernetic elbow prostheses-PartI, PartII," IEEE Trans. Biomed. Eng., vol.37, pp.1025-1047, 1990.

[5] R. J. Triolo and G. D. Moskowitz, PPThe theoretical development of a multichannel time-series myoprocessor for simultaneous limb function detection and muscle force estimation," IEEE Trans. Biomed. Eng., vol. 36, pp. 1004-1017, 1989.

[6] O. Fukuda, T. Tsuji, M. Kaneko and A. Otsuka: "A Human-Assisting Manipulator Teleoperated by EMG Signals and Arm Motions," IEEE Trans. Rob. and Aut., vol. 19, no. 2, pp. 210-222, 2003.

[7] C. Orizio: "Muscle sound: basis of the introduction of a mechanomyographicsignal in muscle studies," Critical Reviews Biomedical Engineering, vol.21, pp.201-243,1993.

[8] T. D. Barry, J. J. Leonard, A. J. Gitter and R. D. Ball: "Acoustic myography as a control signal for an externally powered prosthesis," Arch Phys Med Rehabil, vol.67, pp.267-269, 1986.

[9] J. Silva, W. Heim and T. Chau: "A Self-Contained, MechanomyographyDriven Externally Powered Proshesis," Archives of Physical Medicine and Rehabilitation, vol.86, no.10, pp.2066-2070, 2005.

[10] C. Orizio, R. Perini, and A. Veicsteinas: "Muscular sound and force relationship suring isometric contraction in man," European journal of applied physiology and occupational physiology., vol.58, pp.528-533, 1989.

[11] C. Orizio, R. Perini, B. Diemont and A. Veicsteinas: "Muscle sound and electromyogram spectrum analysis during exhausting contractions in man," European journal of applied physiology and occupational physiology., vol.65, no.1, pp.1-7, 1992.

[12] T. Tsuji, O. Fukuda, H. Ichinobe and M. Kaneko: "A Log-Linearized Gaussian Mixture Network and Its Application to EEG Pattern Classification," IEEE Trans. Syst., Man, and Cybern.-Part C, vol.29, no.1, pp.60-72, 1999. 